\title{
Clinical manifestations of adult patients requiring influenza-associated hospitalization: a prospective multicenter cohort study in Japan via internet surveillance
}

Tadashi Ishida ${ }^{1}$, Masafumi Seki ${ }^{2}$, Kazunori Oishi ${ }^{3}$, Kazuhiro Tateda ${ }^{4}$, Jiro Fujita ${ }^{5}$, Jun-ichi Kadota $^{6}$, Akihiko Kawana ${ }^{7}$, Koichi Izumikawa ${ }^{8}$, Toshiaki Kikuchi ${ }^{9}$, Norio Ohmagari ${ }^{10}$, MItsuhiro Yamada ${ }^{11}$, Takaya Maruyama ${ }^{12}$, Takahiro Takazono ${ }^{13}$, Makoto Miki ${ }^{14}$, Yoshitsugu MIyazaki ${ }^{15}$, Yoshitaka Yamazaki ${ }^{16}$, Hiroshi Kakeya ${ }^{17}$, Kenji Ogawa ${ }^{18}$, Hideaki Nagai $^{19}$, and Akira Watanabe ${ }^{20}$

${ }^{1}$ Kurashiki Central Hospital

${ }^{2}$ Tohoku Medical and Pharmaceutical University

${ }^{3}$ Toyama Institute of Health

${ }^{4}$ Toho University School of Medicine

${ }^{5}$ University of the Ryukyus

${ }^{6}$ Nagasaki Harbor Medical Center

${ }^{7}$ National Defense Medical College

${ }^{8}$ Nagasaki Unviersity Graduate School of Biomedical Sciences

${ }^{9}$ Niigata University Graduate School of Medical and Dental Sciences

${ }^{10}$ National Center for Global Health and Medicine

${ }^{11}$ Tohoku University Graduate School of Medicine

${ }^{12}$ National Hospital Organization Mie National Hospital

${ }^{13}$ Nagasaki University Graduate School of Biomedical Sciences

${ }^{14}$ Japanese Red Cross Sendai Hospital

${ }^{15}$ National Institute of Infectious Diseases

${ }^{16}$ Shinshu Medical Center

${ }^{17}$ Osaka City University Graduate School of Medicine

${ }^{18}$ National Hospital Organization HIgashinagoya National Hospital

${ }^{19}$ National Hospital Organization Tokyo National Hospital

${ }^{20}$ Tohoku Bunka Gakuen University

July 13, 2020

\footnotetext{
Abstract

Background: Influenza remains a clinically heavy burden worldwide. However, evidence about clinical manifestation has been lacking among severely ill patients infected with influenza. Methods: The clinical data for patients who were severely ill with influenza, and required hospitalization were gathered and analyzed between November 2014 and August 2019 (5 influenza seasons) using an internet-surveillance system. Results: A total of 924 patients were enrolled and analyzed. The median age was 78 years (IQR, 67-84), and the patients in the 2015-2016 season were significantly younger than those in other seasons. Pneumonia was the most common disease indicated as a cause for hospitalization, followed by a poor general condition and
} 
exacerbation of underlying respiratory diseases. Antiviral drugs were administered in $97.0 \%$ of the patients with peramivir being the most-frequently use antiviral. In-hospital death was recorded for 44 patients (4.8\%). Multivariate analysis indicated that nursing home resident (OR: 6.554) and obesity (OR: 24.343) were independent predictors of in-hospital mortality. Conclusion: Complications of influenza infection remain a heavy burden especially among the elderly. Continuous nationwide surveillance will be required to grasp the actual situation of influenza epidemics.

\section{Keywords}

influenza, hospitalization, internet-surveillance system, mortality, elderly

\section{INTRODUCTION}

Influenza causes epidemics worldwide every winter and is associated with mortality and morbidity. Influenza presents a wide range of clinical findings from self-limited upper respiratory tract infection to life-threatening illness that includes respiratory failure, encephalopathy, and so on. Influenza-related deaths in Japan are estimated to 3000-10000 every year, and excess death in the elderly is an especially serious problem ${ }^{1}$. Additionally, the emergence of a novel influenza virus following the $2019 \mathrm{H} 1 \mathrm{~N} 1$ pandemic was deeply concerning.

Treatment guidelines for adults with novel influenza were published by the Japanese Ministry of Health, Labour and Welfare in $2014^{2}$. However, in the process of making these guidelines, it was revealed that evidence about clinical manifestation was lacking for severely ill patients with influenza. Thus, the Japanese Respiratory Society (JRS) decided to gather detailed information about severely ill patients with influenza who required hospitalization, with the goal of acquiring evidence that might improve subsequent medical care for influenza cases. The present study, which was the result of that decision, was a multicenter, prospective observational study, for which we built an internet-surveillance system to gather and analyze data from members of the JRS. From the beginning, several core institutions participated in this surveillance as a pilot study. We show the results from 5 consecutive influenza seasons.

\section{METHODS}

\subsection{Patients}

This study enrolled patients older than 15 years of age who were diagnosed with influenza and required hospitalization between November 2014 and August 2019. Formal and collaborating members of the JRS throughout the country were invited to participate in the study. The members logged into the internet-surveillance system ${ }^{3}$ and registered the patients' data. The required data included age, sex, medical professional or not, presence of family transmission, diagnostic methods, type of influenza, the onset date, the hospitalized date, vaccination history for both influenza and pneumococcus, underlying diseases or comorbidity, smoking habits, presence of obesity (body mass index[?]30), reasons for hospitalization, presence of pneumonia, category and types of pneumonia, causative pathogens of pneumonia, antiviral drugs used, presence of oxygen therapy or artificial ventilation, antimicrobials used, and outcomes. The system sent reminder mails 14, 30, and 90 days after registration to follow up on the original registration. The internet surveillance committee of the JRS extracted and analyzed the registered data twice a year.

This study was registered with UMIN (UMIN000015989) and was approved by all participating institutional review boards. All patients gave their informed consents by being given opportunities to refuse to participate.

\subsection{Definitions}

Influenza was diagnosed based on the results of rapid antigen detection test using nasopharyngeal swab or sputum specimen, polymerase chain reaction (PCR) method or clinical diagnosis. Clinical diagnoses were made when those who had contact with confirmed influenza patients revealed typical symptoms compatible with influenza.

Patients with pneumonia were diagnosed based on having new radiographic infiltrate, and were classified as having community-acquired pneumonia (CAP) and nursing and healthcare-associated pneumonia (NHCAP) 
according to the JRS guidelines ${ }^{4}$. The types of pneumonia also were categorized as primary viral pneumonia, secondary bacterial pneumonia, or a mixture of the two based on the judgement of the attending physician. Bacterial causes were identified according to sputum and/or blood culture, urinary antigen test, and other microbiologic investigation.

The influenza season is defined as extending from September to the following August. However, the first season was shorter because we started to record from November 2014.

\subsection{Statistical analysis}

The data was analyzed using the Statistical Analysis System ${ }^{\circledR}$ software program, version 9.4 (SAS Institute, Inc., Cary, NC, U.S.A.). Continuous variables were expressed as medians and interquartile ranges (IQRs). Continuous data from multiple groups were compared using the Mann-Whitney U test with the Holm's correction. A $p$ value $<0.05$ was considered statistically significant.

The risk factors for infection with mortality were analyzed using a logistic regression model. All of the variables with $p<0.10$ in the univariate analysis were included to construct the multivariate analysis. Odds ratios (ORs) and $95 \%$ confidence intervals (CIs) were calculated.

\section{RESULTS}

\subsection{Patient characteristics}

A total of 965 patients were registered between January 2015 and August 2019. The numbers of patients for each season were 167 in 2014-2015, 217 in 2015-2016, 179 in 2016-2017, 298 in 2017-2018, and 105 in 20182019. Thirty patients were excluded because their admissions were not associated with influenza. Another eleven patients also were excluded because the causes of admission were not described. Thus, 924 patients were analyzed. The patient characteristics are shown in Table 1 . The median age was 78 years (IQR, 67-84). The age distribution of each season is indicated in Figure 1. The median ages of each season were 78 years (IQR, 66.5-85.5) in 2014-2015, 70 (55-80) in 2015-2016, 80.5 (73-86) in 2016-2017, 79 (70-86) in 2017-2018, and 74.5 (63.5-81) in 2018-2019. The patients in the 2015-2016 season were significantly younger than those in other seasons $(\mathrm{p}<0.05)$.

A total of 684 patients $(74.0 \%)$ had influenza A, and 237 patients $(25.6 \%)$ had influenza B. Twenty-five patients $(2.7 \%)$ were positive for both influenza $\mathrm{A}$ and $\mathrm{B}$, and the influenza type was unknown in 28 patients (3.0\%) who were diagnosed on clinical basis. Two hundred and twenty-two (24.0\%) and 95 patients (10.3\%) had been immunized with an influenza vaccine or the 23 -valent pneumococcal polysaccharide vaccines, respectively.

\subsection{Causes of hospitalization}

Table 2 indicates the causes of hospitalization for all patients. Pneumonia was the most common cause representing $55 \%$ of all cases. The breakdown of pneumonia category was community-acquired (44.3\%), nursing and healthcare-associated (10.2\%), and hospital-acquired (0.5\%). The second-most common cause $(27.3 \%)$ was a poor general condition due to the influenza infection itself. Other causes include exacerbation of underlying chronic respiratory disease (chronic obstructive pulmonary disease [COPD], interstitial pneumonia, bronchial asthma, and so on), heart failure, renal failure, and miscellaneous. Rhabdomyolysis and encephalopathy were recognized in $1.0 \%$ and $0.6 \%$, respectively. Thirty-nine patients $(4.2 \%)$ were admitted due to social indication (the very elderly, those living alone, or those unable to reach medical facilities easily).

\subsection{Pneumonia characteristics}

The types of pneumonia categorized were primary viral pneumonia in 39 patients $(7.6 \%$ of all pneumonia cases), secondary bacterial pneumonia in 198 patients (38.7\%), and a mixture of both in 230 patients (45.0\%). Forty-four cases $(8.6 \%)$ were judged as unclassified.

In patients with secondary bacterial pneumonia and mixture pneumonia, the microbiological etiology was determined in 156 cases (36.4\%). The distribution was as indicated in Table 3. Streptococcus pneumoniae 
was the most common pathogen (89 cases), followed byHaemophilus influenzae (27) and Staphylococcus aureus (20); six of the 20 S. aureus strains were methicillin-resistant.

\subsection{Treatment}

Antiviral drugs were administered in 893 patients (97.0\%). The breakdown of the drugs was peramivir in 529 patients, oseltamivir in 217 , laninamivir in 87 , baloxavir in 12 , zanamivir in 4 , oseltamivir + peramivir in 26 , laninamivir + peramivir in 13 , zanamivir + peramivir in 2 , baloxavir + peramivir in 1 , and oseltamivir + zanamivir in 1 .

Antimicrobials were administered in 637 patients $(68.9 \%)$. The most common antimicrobials used were ceftriaxone and sulbactam/ampicillin, consistent with the guidelines for CAP ${ }^{4}$. Oxygen was required in 531 cases $(57.5 \%)$, and mechanical ventilation including non-invasive positive pressure ventilation (NPPV) was performed in 84 cases $(9.1 \%)$. There was no case in which extracorporeal membrane oxygenation (ECMO) was performed during the study period.

\subsection{Mortality}

In-hospital death was recorded for 44 patients (4.8\%). The median age for the 44 deaths was 82 (IQR 66.588.5). Among the mortalities, 26 were male patients. The causes of hospitalization were pneumonia in 28 cases, poor general status in 11, heart failure in 2, renal failure in 2, and acute exacerbation of COPD in 1. One patient was transferred to the hospital in a state of cardiopulmonary arrest; for this patient, the actual cause of death was unknown.

Univariate analysis identified two variables (nursing home resident and obesity) with $p$ values $<0.10$; these variables were incorporated into the multivariate analysis. Both of these factors were thought to be independent predictors of in-hospital mortality (Table 4): nursing home resident yielded an OR 6.554, 95\% CI 2.663-16.127, and $p<0.0001$, while obesity yielded an OR 24.343, 95\% CI 3.331-177.885, and $p=0.017$.

\section{DISCUSSION}

Influenza remains a heavy clinical burden worldwide. Recent reports have suggested estimated annual hospitalization rates in various countries varying from 13 to 141 per 100,000 population ${ }^{5-10}$. In Japan, $1.0 \%$ of influenza-positive patients aged under 75 years were hospitalized between 2012 and $2016{ }^{11}$. However, those reports included pediatric patients, and focused mainly on respiratory complications such as pneumonia.

The JRS conducted a surveillance of adults and adolescent patients with influenza requiring hospitalization. All causes for hospitalization related to influenza infection were enrolled. For this study, we introduced a new surveillance system that worked via the internet. By using this internet-based system, prospective real-time data can be registered and renewed rapidly from many institutions throughout Japan, and reminder mails can be sent.

In our study, most cases of influenza-related hospitalization in Japan occurred in the elderly, as has been previously in papers from various countries ${ }^{7,10,12-14}$. However, the age distribution during the 2015-2016 season was significantly younger than those of other seasons. This distribution was thought to reflect the fact that the predominant influenza virus strain in the 2015-2016 season was A (H1N1) pdm. This observation confirms a previous report indicating that the median age of hospitalized cases in the 2009 A (H1N1) pdm pandemic was younger than is typically seen for seasonal influenza ${ }^{14}$. A considerable number of younger adults who were naïve for A (H1N1) pdm in the preceding seasons might have been infected for the first time during the 2015-2016 season, and some of these individuals presumably were hospitalized due to complications.

The most common cause of hospitalization was pneumonia, an observation that is consistent with the results of another Japanese study by Maruyama and colleagues ${ }^{15}$. Primary viral pneumonia was recognized in only $7.6 \%$ of all pneumonia cases. However, $68 \%$ of those primary viral pneumonia cases were reported in the 2015-2016 season. This pattern also is thought to be attributable to the predominance of A (H1N1) in the 2015-2016 season. Diagnosis of primary viral pneumonia seems to depend on attending physicians' decisions based on clinical findings. However, some cases were diagnosed via the PCR method using bronchoalveolar 
lavage fluid (BALF). Recently, a multiplex real-time PCR method has been introduced for diagnosis of $\mathrm{CAP}^{16}$, which might lead to an apparent increase in the frequency of influenza viral pneumonia. In the present study, the major pathogens in secondary bacterial pneumonia were S. pneumoniae, H. influenzae, and $S$. aureus consistent with previous report ${ }^{17}$. However, none of these bacterial species appeared to be an independent risk factor for mortality.

The second-most common cause of hospitalization in our study was "poor general condition". Most of our patients were elderly people who had multiple medical conditions, and thus were fragile in the face of influenza infection. In Japan, the threshold of hospitalization generally is lower than in other countries. Therefore, in Japan, the elderly were more typically hospitalized when suffering from influenza.

Our study enrolled patients over 15 years old. Thus, there were few cases of encephalopathy with influenza. Additionally, our cases included few pregnant women, though such patients have been defined as having a high risk of complication from influenza ${ }^{18}$. In Japan, there have not been many severe cases of pregnant women with influenza, and no fatal cases of pregnant women with influenza were reported during the pandemic of 2009 A (H1N1) ${ }^{19}$.

Most patients (97\%) received antiviral therapy from the early stage of the illness in this study. In Japan, those who are suspected to have influenza undergo rapid diagnostic antigen testing, and antiviral therapy is initiated for those with positive results or on a clinical basis as a standardized strategy ${ }^{20,21}$. Peramivir was the most-commonly used antiviral agent because this medication is provided as an injection drug, a format that is useful for treatment of inpatients. In Japan, baloxavir marboxil, a novel cap-dependent endonuclease inhibitor, was introduced to the market in March, 2018. An effect of baloxavir marboxil is expected because this medication has an antiviral mechanism that is distinct from that of neuraminidase inhibitors (NAIs) 22,23 . However, there is some concern that viral strains with reduced baloxzavir susceptibility will emerge especially in younger children, seriously ill patients, or the immunocompromised ${ }^{24}$. Few patients underwent antiviral therapy with baloxavir in the present study. It will be necessary to monitor the future trends of baloxavir use. Treatment of hospitalized patients with the combination of barloxavir and NAIs has been reported to be effective ${ }^{25}$. This combination treatment is thought to be a possible strategy that deserves further examination in future studies.

In total, $4.8 \%$ of the hospitalized cases were fatal. This rate was lower than that reported in other countries $8,14,26,27$. The lower value was thought to reflect the generally lower threshold for hospitalization in Japan compared to other countries. As mentioned above, early diagnosis and treatment are the essential strategies against influenza in Japan. In our study, pneumonia was overwhelmingly the leading cause of death associated with influenza as reported previously $8,11,12,14,15$. Secondary bacterial pneumonia following influenza infection remains a serious problem, especially in the elderly. Our multivariate analysis proved that nursing home resident and obesity were independent risk factors for mortality. Obesity has been identified as a prognostic factor for influenza ${ }^{28.29}$. Recently, the number of obese people has increased in Japan, which has lead in turn to several clinical problems including increased susceptibility to infection.

This study had several limitations. First, the number of participating institutions was small because this research was conducted as a pilot study. Moreover, about one third of the enrolled cases came from a single hospital (Kurashiki Central Hospital), which might lead to institutional bias. Second, the scale of the participating institutions varied from primary physician's clinics to educational hospitals; thus, clinical levels were not uniform. Sufficient examinations could not be done in all of the cases. The detailed data obtained from a single center will be reported as a supplement to this report. Third, the influence of vaccination could not be analyzed because vaccination status was not obtained for many patients despite of a prospective nature of this study. Fourth, detailed clinical data for each case were not obtained because of the limits of the internet-surveillance system.

In conclusion, complications of influenza infection remain a heavy burden especially among the elderly. Continuous nationwide surveillance will be required to clarify the actual situation of influenza.

\section{AUTHORS CONTRIBUTIONS}


Tadashi Ishida: writing-original draft (lead); conceptualization; data curation. Masafumi Seki:conceptualization; data curation; writing-review and editing.Kazunori Oishi: conceptualization (lead); writing-review and editing. Kazuhiro Tateda: conceptualization; data curation; writing-review and editing.

Jiro Fujita: conceptualization; data curation; writing-review and editing. Jun-ichi Kadota: conceptualization; data curation; writing-review and editing. Akihiko Kawana: conceptualization; data curation; writing-review and editing. Koichi Izumikawa:conceptualization; data curation; writing-review and editing.Toshiaki Kikuchi: conceptualization; data curation; writing-review and editing. Norio Ohmagari: conceptualization; data curation; writing-review and editing. Mitsuhiro Yamada:conceptualization; data curation; writing-review and editing. Takaya Maruyama: conceptualization; data curation; writing-review and editing. Takahiro Takazono: data curation; writing-review and editing. Makoto Miki: writing-review and editing. Yoshitsugu Miyazaki: writing-review and editing.Yoshitaka Yamazaki: writing-review and editing. Hiroshi Kakeya: writing-review and editing. Kenji Ogawa:writing-review and editing. Hideaki Nagai: writing-review and editing. Akira Watanabe: conceptualization; data curation; writing-review and editing.

\section{ORCID ID}

Tadashi Ishida: https://orcid.org/0000-0001-9628-9125

\section{REFERENCES}

1. National Institute of Infectious Diseases. [in Japanese] Infect Agents Surveil Report. 2019; 40: 192-194.

2. The Ministry of Health, Labour and Welfare. The treatment guidelines for adults of novel influenza. https://www. mhlw. go.jp/seisakunitsuite/bunya/kenkou_iryou/kenkou/kekkakukansenshou/infulenza/dl/guideline. pdf Accessed June 6, 2020.

3. The Japanese Respiratory Society. The influenza internet surveillance system. [in Japanese] https: //www. jrs. or. jp/iis/ Accessed June 6, 2020.

4. The Japanese Respiratory Society. The JRS guidelines for management of pneumonia in adults. [in Japanese] The Japanese Respiratory Society, Tokyo, 2017.

5. Abdel-Hady, Balushi, RM, Abri BA, et al. Estimating the burden of influenza-associated hospitalization and death in Oman (2012-2015). Influenza Other Respir Viruses 2018; 15: 146-152.

6. Palekar RS, Rolfes MA, Arriola CS, et al. Burden of influenza-associated respiratory hospitalizations in Americas, 2010-2015. PloS One 2019 Sep 6; 14(9): 0221479. doi: 10.1371/journal.pone.0221479.

7. Ng Y, Chua LAV, Ma S, Lee VJM. Estimates of influenza-associated hospitalizations in tropical Singapore, 2010-2017: higher burden estimated in more recent years. Influenza Other Respir Viruses 2019; 13: $574-581$.

8. Ao T, McCracken JP, Lopez MR, et al. Hospitalization nd death among patients with influenza, Guatemala, 2008-2012. BMC Public Health 2019 May 10; 19 (Suppl 3):463. doi: 10.1186/s12889-0196781-6.

9. Pivette M, Nicolay N, de Lauzun V, Hubert B. Characteristics of hospitalization with an influenza diagnosis, France, 2012-2013 to 2016-2017 influenza seasons. Influenza Other Respir Viruses 2020; 14: 340-348.

10. Moa AM, Muscatello DJ, Turner RM, Maclntyre CR. Estimated hospitalizations attributable to seasonal and pandemic influenza in Australia: 2001-2013. PloS One 2020 Apr 13; 15(4):e0230705. doi: 10.1371/journal.pone.0230705.

11. Yokomichi H, Mochizuki M, Lee JJ, Kojima R, Yokoyama T, Yamagata Z. Incidence of hospitalization for severe complications of influenza virus infection in Japanese patients between 2012 and 2016: a crosssectional study using routinely collected administrative data. BMJ Open 2019 Jan 17; 9(1):e024687. doi: 10.1136/bmjopen-2018-024687.

12. Thompson WW, Shay DK, Weintraub E, et al. Influenza-associated hospitalizations in the United States. JAMA 2004; 292: 1333-1340.

13. Shrestha SS, Swerdlow DL, Borse RH, et al. Estimating the burden of 2009 pandemic influenza A (H1N1) in the United States (April 2009-April 2010). Clin Infect Dis 2011; 52: S75-S82. 
14. Louie JK, Acosta M, Winter K, et al. Factors associated with death or hospitalization due to pandemic 2009 influenza A(H1N1) infection in California. JAMA 2009; 302: 1896-1902.

15. Maruyama T, Fujisawa T, Suga s, et al. Outcome and prognostic features of patients with influenza requiring hospitalization and receiving early antiviral therapy. Chest 2016; 149: 526-534.

16. Gadsby NJ, Russell CD, McHugh MP, et al. Comprehensive molecular testing for respiratory pathogens in community-acquired pneumonia. Clin Infect Dis 2016; 62: 817-823.

17. Klein EY, Monteforte B, Gupta A, et al. The frequency of influenza and bacterial coinfection: a systematic review and meta-analysis. Influenza Other Respir Viruses 2016; 10: 394-403.

18. Uyeki TM, Bernstein HH, Bradley JS, et al. Clinical practice guidelines by the Infectious Diseases Society of America: 2018 update on diagnosis, treatment, chemoprophylaxis, and institutional outbreak management of seasonal influenza. Clin Infect Dis 2019; 68: e1-e47.

19. The Ministry of Health, Labour and Welfare. Age distribution of fatal cases dueb to 2009 pandemic influenza A (H1N1) in Japan https: //www. mhlw. go. jp/bunya/kenkou/kekkaku-kansenshou04/ rireki/100331-03. html Accessed June 6, 2020.

20. Sugaya N, Shinjoh M, Mitamura K, et al. Comparison of the clinical effectiveness of oseltamivir and zanamivir against influenza in children. Clin Infect Dis 2008; 47: 339-345.

21. Sugaya N. Widespread use of neuraminidase inhibitors in Japan. J Infect Chemother 2011; 17: 595-601.

22. Hayden FG, Sugaya N, Hirotsu N, et al. Baloxavir marboxil for uncomplicated influenza in adults and adolescents. 2018; 379: 913-923.

23. Ison MG, Postmouth S, Yoshida Y, et al. Early treatment with baloxavir marboxil in high-risk adolescent and adult outpatients with uncomplicated influenza (CAPSTONE-2): a randomised, placebocontrolled, phase 3 trial. Lancet Infect Dis 2020 Jun 8; S1473-3099(20)30004-9. doi: 10.1016/S14733099(20)30004-9.

24. Uehara T, Hayden FG, Kawaguchi K, et al. Treatment-emergent influenza variant viruses with reduced baloxavir susceptibility: impact on clinical and virologic outcomes in uncomplicated influenza. J Infect Dis 2020; 221: 346-355.

25. Yoshimura Y, Sasaki H, Horiuchi H, et al. Early combination treatment with baloxavir and peramivir for hospitalized adults with influenza A in Yokohama, Japan. Eur J Clin Microbiol Infect Dis 2020 Apr 14. doi: 10.1007/s10096-020-03888-7.

26. Schanzer DL, Langley JM, Tam TW. Co-morbidities associated with influenza-attributed mortality, 1994-2000, Canada. Vaccine 2008; 26: 4697-4703.

27. Lee N, Choi KW, Chan PK, et al. Outcomes of adults hospitalised with severe influenza. Thorax 2010; 65: 510-515.

28. Louie JK, Acosta M, Samuel MC, et al. A novel risk factor for a novel virus: obesity and 2009 pandemic influenza A (H1N1). Clin Infect Dis 2011; 52: 301-312.

29. Moser JS, Galindo-Fraga A, Ortiz-Hernández AA, et al. Underweight, overweight, and obesity as independent risk factors for hospitalization in adults and children from influenza and other respiratory viruses. Influenza Other Respir Viruses 2019; 13: 3-9.

\section{FIGURE 1. The age distribution of cases by season.}

\section{Hosted file}

Figure 1 .pptx available at https://authorea.com/users/341877/articles/468771-clinicalmanifestations-of-adult-patients-requiring-influenza-associated-hospitalization-aprospective-multicenter-cohort-study-in-japan-via-internet-surveillance

\section{Hosted file}

Tables.docx available at https://authorea.com/users/341877/articles/468771-clinicalmanifestations-of-adult-patients-requiring-influenza-associated-hospitalization-a-

prospective-multicenter-cohort-study-in-japan-via-internet-surveillance 